\title{
Влияние систем менеджмента качества на финансовые показатели компаний: концептуальный подход
}

\author{
Багаев А.C. ${ }^{11}$, Кочугуева M.H. ${ }^{12}$
}

Целью настоящей статьи является систематизащия классических подходов в области систем менеджмента качества и определение возможности их применения к российским компаниям. Много внимания уделяется описанию различий между традиционным подходом $\kappa$ качеству и идеями TQM, как с идеологчческой точки зрения, так и с точки зрения применяемых процедур учета. На примере наиболее известных подходов и авторов (Джуран, Деминг, Кросби) показана динамика развития идеологии управления качеством. Предлагаются возможные механизмы влияния менеджмента качества на конкретные показатели деятельности компаний, что позволяет сформулировать возможные иели $u$ методологию эмпирических исследований. Рассматриваются перспективы развития идей качества в России, описываются организационные предпосылки, необходимые для того, чтобы российские компании смогли перейти $к$ эффективному внедрению систем менеджмента качества.

\section{JEL: $L 15$}

Ключевые слова: менеджмент качества, процессный подход, эффективность работь компании, стоимость компании, всеобщее управление качеством

На сегодняшний день тезис о том, что качество является серьезным конкурентным преимуществом, не ставится под сомнение практически никем, компании полагают качество значительным элементом своей стратегии. В связи с этим особенно важно исследовать влияние подобных идей и наличие систем менеджмента качества на конкретные финансовые показатели.

Дискуссии вокруг вопросов качества не утихают до сих пор: с одной стороны, продолжаются теоретические поиски на базе идей Э. Деминга, Дж. Джурана и Ф. Кросби, с другой - современная экономическая наука требует эмпирических исследований о влиянии новых подходов к управлению качеством на эффективность работы компаний.

Слово «качество» следует понимать в широком смысле, относя его не только к выпускаемой продукции: это также характеристика маркетинга, послепродажного обслуживания, наконец - характеристика бизнес-процессов внутри компании. Например, сертификация по стандартам ИСО 9000 может являться подтверждением соответствия общепринятым стандартам системы менеджмента качества в этом широком смысле системы контроля, охватывающей практически всю деятельность данной компании. Очевидно, что такая система должна оказывать влияние на ряд финансовых и нефинансовых показателей компании, что является предметом эмпирических исследований в этой области: на самом ли деле сертификация (ИСО 9000) является благом для компании, каковы ее положительные эффекты, каков их механизм?

Существующие работы по этой теме весьма многочисленны, но имеют один серьезный недостаток: как правило, они представляют собой описание удачных практик сертификации, то есть это по сути работы типа «саse study». Очевидно, что рассмотрение только «хороших» историй не может дать полной картины изучаемого явления. Существуют также работы,

\footnotetext{
11 Экономист, ОАО «ВТБ Лизинг»; выпускник магистерской программы «Стратегическое управление финансами фирмы» факультета экономики НИУ ВШЭ.

12 Доцент кафедры экономики и финансов фирмы факультета экономики НИУ ВШЭ, канд. эконом. наук, доцент.
} 
делающие акцент на понимании динамики «естественных» (нефинансовых) показателей качества, таких, как качество продукции, удовлетворенность потребителя, количество жалоб и других, связанных с сертификацией. Недостатком этих работ является то, что данные о такого рода показателях сообщаются самими компаниями и, следовательно, могут быть смещенными. Кроме того, связь «естественных» показателей с финансовыми неочевидна, и потому описать влияние сертификации на финансовые аспекты деятельности не всегда удается.

Существует очень небольшое число работ, пытающихся напрямую увязать финансовые показатели и факт сертификации компании по стандартам ИСО 9000. В отношении российских компаний не было проведено ни одного серьезного исследования. Поскольку количество сертифицированных компаний в нашей стране растет, проведение подобного исследования является целесообразным и весьма актуальным. Оно поможет выявить, какие мотивы движут российскими компаниями при сертификации, и, следовательно, дать оценку отношения бизнеса к управлению качеством. Кроме того, наличие или отсутствие связи сертификации и финансовых показателей позволит сделать определенные выводы о перспективах и применимости идеи всеобщего управления качеством в нашей стране.

Важно также исследовать связь между сертификацией и изменением стоимости компании: ведь если принято решение о сертификации а, значит, вероятно, и переходе к следованию парадигме всеобщего управления качеством, необходимо оценить его влияние на благополучие акционеров. Существует мнение, что сертификация сама по себе не добавляет стоимости компании, поскольку не привносит ничего нового, поэтому рассмотрение вопросов, связанных с капитализацией, становится особенно важным. Необходимо понять взаимосвязь между сертификацией и следованием компании идеалам TQM, исследовать, влияет ли сертификация на рост стоимости компании, и сделать вывод о стратегической оправданности этого шага.

Управление качеством является неотъемлемой составляющей менеджмента организации и определяется в стандартах ISO 9000 (2000) как «скоординированная деятельность по руководству и управлению организацией применительно к качеству» Управление качеством - это не минимизация дефектов, а стратегический ориентир компании, направленный на постоянное поддержание и улучшение качества. Под «системой менеджмента качества» понимается распространение идеи поддержания и улучшения качества на все элементы цепочки ценностей, а также за ее пределы (связи с поставщиками и потребителями). Отношение к качеству как к стратегическому ориентиру компании называют «Total quality management» (TQM).

Последний тезис является удобной отправной точкой для систематизации классических взглядов, которые легли в основу научной парадигмы качества. Мы предлагаем ориентироваться на базовые идеи уже упомянутых гуру в области качества, поскольку предложенные ими идеи не потеряли своей актуальности и могут быть полезны для каждого из этапов внедрения идей TQM в организации.

Всегда, когда речь идет об управлении качеством, неизбежно возникает вопрос о затратах на качество. Один из первых исследователей в данной области Дж. Джуран предложил еще в 70-х годах прошлого столетия принципиально новый подход для анализа затрат на качество. Идея заключалась в том, чтобы разделить стоимость затрат на качество на четыре категории: затраты на контроль качества (состоящие из затрат на предотвращение дефектов и затрат на оценку) и затраты на фактический брак (состоящие из внешних и внутренних). Новация состоит в том, что в рамках традиционной парадигмы затраты на качество не являются предметом отдельного анализа. Так, в управленческом учете затраты на брак как правило относятся на себестоимость качественной продукции, увеличивая ее. Недостаток подобного подхода заключается в «размывании» эффекта брака; кроме того, как правило, имеется нормативная норма отбраковки, которая, с одной стороны, позволяет удерживать некоторые показатели в определенных рамках, а с другой, узаконивает определенный процесс дефектов. Традиционный подход ориентирован на достижение 
нормативного уровня, а не на постоянное улучшение качества, так как предоставляет своего рода контрольный аппарат, но никак не аналитический.

Деление же затрат на категории, предложенное Джураном, является в первую очередь инструментом анализа. Джуран настаивает на ведении особой отчетности по качеству, которая должна учитываться руководством при принятии решений. При этом помимо аналитического инструмента данный подход указывает некоторое направление для развития: предполагается, что затраты на брак уменьшаются с ростом качества, а затраты на контроль - увеличиваются. Суперпозиция этих величин дает кривую с минимумом, который представляет собой оптимальный уровень качества; к нему, в идеале, компании и надо стремиться. Каждый элемент данной концепции интуитивно понятен: затраты на брак падают с уменьшением количества брака, но затраты на контроль, необходимый для обеспечения такого уровня качества, по идее, должны расти. Стратегические выгоды такого подхода также очевидны: снижение затрат на качество означает снижение общих затрат компании, что усиливает ее конкурентную позицию. Еще раз подчеркнем, что затраты на качество можно «отделить от себестоимости» и анализировать «отдельно». Следует напомнить, что Джуран возлагает функции выделения и анализа затрат на качество на некий специальный отдел по контролю качества, выполняющий контрольную и аналитическую работу и определяющий возможные пути сокращения этого вида затрат.

Здесь возникает вполне справедливое замечание: если данный подход подразумевает некий минимум функции затрат на качество, то в чем же отличие от традиционной парадигмы? Ведь минимальная сумма затрат, отнесенная на себестоимость, есть та же самая узаконенная норма брака. Может показаться, что здесь нет ничего качественно нового!

И тем не менее это не так. Во-первых, Джуран дает управленцам в руки аналитический инструмент, о котором раньше никто и не думал, что само по себе позволяет снизить допуск на брак. Во-вторых, никто не говорит, что минимум затрат на качество достигается при некотором проценте брака: он может достигаться и при «идеальном» производстве, при котором нет брака вовсе! Действительно, минимум функции вполне может лежать правее точки стопроцентного качества, и тогда условный экстремум будет достигаться на правой границе отрезка, соответствующей бездефектному производству. Эта идея соответствует следующей ключевой точке истории ТQМ, а именно идеям Эдвардса Деминга.

Деминг разработал свои основные положения живя в Америке, но там они не нашли признания, в отличие от Японии, где были с успехом внедрены. Можно сказать, что именно идеология, разработанная этим ученым, вывела ряд японских компаний на мировой уровень, дав невообразимую по тем временам конкурентоспособность. Именно в Японии была учреждена премия имени Деминга, вручаемая компаниям за качество и надежность выпускаемой ими продукции.

В чем же заключается эта чудесная идея, которая, как показала история, способна творить чудеса? Деминг идет гораздо дальше Джурана и заявляет, что приемлемого уровня брака не существует и не может существовать: единственно допустимый уровень брака, к которому может стремиться компания, - ноль.

Аргументация здесь такова: невыполнение требований по качеству означает потерю компанией репутации среди клиентов, а это приводит к крайне высоким затратам, которые значительно превосходят затраты на устранение обнаруженных дефектов. Следовательно, если компания признает наличие допустимого уровня брака, то она не понимает в полной мере проблемы потери репутации, и качество не является ее стратегической задачей, в противном случае компания может стремиться только к отсутствию дефектов. Напомним, что к такому выводу можно прийти, рассматривая и идеи Джурана, - но аргументации здесь коренным образом различаются.

В самом деле, в то время как Джуран возлагает функции по контролю качества и анализу соответствующих затрат на специальный отдел, Деминг отрицает необходимость специальной отчетности по качеству. Вернее, он признает необходимость статистического контроля - но в натуральных показателях, таких, как процент брака. Вообще, существуют 
так называемые «четырнадцать принципов управления качеством Деминга», которые отражают его философию в отношении качества, но нас интересуют лишь основные идеи. В частности, помимо того что было отмечено выше, Деминг настаивает на том, чтобы качество являлось стратегическим приоритетом фирмы в целом - то есть не только ее руководства, но также и каждого ее сотрудника. Этому должна способствовать коммуникация между подразделениями, которая зачастую затруднена даже в наше время. Каждый сотрудник должен иметь возможность - и стимул, что не менее важно, - к самосовершенствованию.

Все это звучит утопично, но практика показала, что реализация этих принципов способна творить чудеса. При этом нет ничего удивительного, что эта идеология сработала именно в Японии, поскольку японский менталитет как нельзя лучше соответствует идеалам Деминга: японцы с определенной долей фанатизма относятся к работе, и наличие некой «идеальной» цели, как то постоянное повышение качества, является для них значительным стимулом.

Вообще говоря, идеология всеобщего вовлечения в качество является, пожалуй, самой важной для сегодняшних реалий нашей страны, а ее утопичность, видимый контраст с действительным положением вещей позволяют, с одной стороны, подчеркнуть недостатки принципов работы сегодняшних организаций, с другой - лучше разглядеть пути улучшения, выхода из сложившейся ситуации. Так, чувство коллективной ответственности за качество, разумеется, нельзя навязать - но зная, что в идеале цель именно такова, можно понять, по крайней мере, направление движения, которое позволит постепенно принять эту идеологию.

Так мы постепенно подходим к идее «качество - процесс»: рассмотрение идей качества в таком ключе возвращает нас к идеям Филипа Кросби. Позицию его, наверное, можно представить как нечто среднее между идеями Деминга и Джурана. Если же избежать употребления термина «средний» и несколько конкретизировать последнее утверждение, то получится следующая формула: Кросби принимает инструментарий Джурана, идеал стопроцентного качества Деминга и дополняет их своим идеологическим наполнением.

Для нас наиболее важно это самое «наполнение», а точнее уже озвученная идея «качество - процесс»: должно эволюционировать отношение к качеству и понимание проблем, связанных с ним.

При этом не следует думать, что данный подход чисто идеологический: слово «понимание» здесь носит не абстрактный, а конкретный характер, поскольку Кросби указывает, с чем, по его мнению, связаны проблемы с качеством. Он производит декомпозицию этого понятия, считая некорректной саму формулировку - «проблема качества»: проблемы могут возникать в ходе разработки изделия или услуги, в процессе производства, при выполнении определенных операций. Таким образом, в идеях Кросби четко просматривается процессный подход, который на сегодняшний день признается одной из центральный идей систем управления качеством.

Не надо думать, что идея эволюции качества у Кросби дублирует идеи Деминга о «постоянном улучшении»: если Деминг предлагает стремление к качеству ради качества же, то Кросби настаивает на идейной эволюции. Эта идеология как нельзя лучше подходит для современных российских компаний, поскольку выдвигает на передний план не только результат - но и процесс. В рамках такой идеологии проще начать движение с нуля - a именно в таком состоянии находится менеджмент качества очень и очень многих российских компаний.

Изложенные три позиции следует рассматривать как историю развития идей TQM, a точнее - краеугольные камни, которые определили ее. Основные идеи, которые лежат в основе того, что сегодня принято называть парадигмой качества, берут начало именно в этих работах: в этом смысле их рассмотрение есть рассмотрение истории парадигмы - не столько как некой хронологии, сколько как источника концептуальных идей каждая из которых согласуется с определенной стадией внедрения философии ТQМ на предприятии. Идеи, которые объединяют эти теории, и определяют сегодняшнюю философию качества. При этом надо понимать, что в «классических» подходах существует множество принципиальных 
расхождений: так, например, Деминг настаивает на преобразовании корпоративной культуры для достижения необходимого качества, в то время как система Джурана работает в рамках традиционной культуры и не требует ее изменения. Поскольку корпоративная культура на сегодняшний момент представляется важным элементом философии качества, то нельзя обойти ее вниманием при описании современного положения вещей.

На самом деле, организационные предпосылки являются неотъемлемой частью парадигмы TQM. Важно заметить, что проблемы с качеством, характерные для российских компаний, вполне могут быть вызваны отсутствием организационных предпосылок, а, следовательно, упрощенным пониманием идеи ТQM и неэффективным управлением качеством.

Да, системы контроля качества, построенные на принципах ОТК, и плановое управление, имевшее место на крупных предприятиях до 90-х годов прошлого века, позволяли обеспечить достаточно высокое качество, но за счет наличия блестящих управленцев, четко понимающих возможные проблемы производственных процессов. На сегодняшний день процессы подчас настолько сложны, что одному человеку разобраться во всем практически невозможно. Именно отсюда следует важнейшая предпосылка TQM: ответственен за качество каждый - и работать над повышением качества тоже должен каждый. Именно этот тезис никак не согласуется с управленческой культурой, сложившейся сегодня в России. В этом смысле Россия - это антииллюстрация к требованию Деминга о том, что работники не должны испытывать чувства страха за свою судьбу, чтобы от них можно было ожидать творческого подхода. У нас же, особенно на сегодняшний день, страх увольнения подчас так велик, что заставляет выполнять указания дословно, не тратя времени на размышления. В такой ситуации большинству работников компании просто не до повышения качества. Конечно, работодатель может устранить этот негативный фактор, влияя на настроения в компании. Тем не менее для сведения к минимуму этого влияния в масштабе всей страны необходимо так или иначе гарантировать работникам их права, возможно, пересмотреть соответствующее законодательство. Несмотря на это, нужно подчеркнуть, что хотя и существует проблема несоответствия общего положения дел в России и идей TQM, в отдельно взятой компании теоретически возможно создать необходимые организационные предпосылки для следования идеям качества.

Всеобъемлющее чувство страха и неуверенность в завтрашнем дне выражаются в том числе и в том, что работники противятся практически любым изменениям в компании. Это значит, что к внедрению идей качества надо подходить осторожно, стараясь не вызвать антипатию со стороны сотрудников компании, - именно такой подход может дать шансы на успех.

Что касается исследователя-практика, то рассуждения об организационных предпосылках могут быть ему не так интересны: его могут заинтересовать такие вещи, как, например, конкретная реакция рынка на наличие системы менеджмента качества или влияние таких систем на показатели деятельности компаний, или влияние систем менеджмента качества на достижение долгосрочного конкурентного преимущества. В то же время надо понимать, что знак влияния СМК на перечисленные параметры определяется именно общим фоном на рынке - по сути, уровнем доверия к менеджменту качества, который, в свою очередь, сильно зависит от уровня отклонения существуюей организационной культуры от идеалов, предписываемых рамках TQM.

Нами было проведено практическое исследование, подробное обсуждение которого находится за рамками данной статьи. Его результат является, казалось бы, несколько парадоксальным: компании, имеющие СМК, статистически более рентабельны, но не демонстрируют более высоких показателей динамики стоимости компании. Вообще говоря, при прочих равных параметрах деятельности компании (методология исследования была подобрана так, чтобы это требование максимально выполнялось) результат об улучшении эффективности предполагает рост стоимости. Тем не менее нельзя забывать, что капитализация определяется не только и не столько фундаментальными показателями 
компании, сколько ожиданиями участников рынка относительно ее действий и их потенциальных результатов. Результат, не согласующийся с тезисом о внутреннем улучшении, говорит о том, что рынок, по крайней мере, проявляет какую-то реакцию на внедрение СМК как некоторое особенное событие, причем эта реакция, вероятно, негативная. Действительно, если бы это было не так, улучшение фундаментальных показателей должно было бы привести к росту стоимости, а этого, как мы видим, не происходит. Конечно, это может быть связано и с тем, что эта информация инкорпорируется в рыночную стоимость гораздо медленнее, чем, скажем, информация о получении сертификата СМК, о котором компания громко заявляет - и в чем нельзя усомниться. Тем не менее, нельзя забывать о том, что динамика рыночной стоимости также отражает текущие настроения участников рынка, которые вовсе не обязательно связаны с фундаментальными показателями типа выручки или рентабельности. Так, набирает популярность позиция, что сертификат - лишь бумажка, получение которой дорого стоит, а значит, получение сертификата никак не увеличивает, а возможно, и уменьшает капитализацию. Отсутствие положительной реакции рынка показывает, что его участники, возможно, придерживаются указанной позиции. Поскольку исследование показывает действительный рост фундаментальных показателей, можно достаточно определенно утверждать, что отсутствие увеличения капитализации связано именно с настроениями рынка. При этом важно то, что реакция рынка - все же не отрицательная, то есть, скорее всего, существует определенное недоверие к роли сертификации в улучшении показателей деятельности компании, но это именно недоверие, а не глубокая антипатия. Можно предположить, что участники рынка здраво оценивают взаимосвязь динамики показателей компании и наличие сертификации и рано или поздно оценят положительные эффекты сертификации, если, конечно, они «проявятся» для данной компании. Если принять идею о целесообразности проведения сертификации и понимать ее связь с идеями TQM, то можно ожидать, что следование идеям качества и создание систем менеджмента качества - это на сегодняшний день здравое стратегическое решение, пусть и не приводящее к скорому росту благосостояния акционеров, но здраво оценивающееся рынком и не наносящее удара по капитализации.

Выводы, озвученные выше, мы приводим в основном в качестве некоего примера, для того чтобы показать, что исследования, связанные с системами менеджмента качества вполне могут дать практические результаты. Несмотря на то что TQM в значительной мере является лишь теоретической концепцией, которая может быть реализована множеством различных способов, практическое следование классическим идеям, упомянутым в настоящей статье, способно продемонстрировать реальное влияние СМК как на показатели деятельности компании, так и на ее стоимость. Это значит, что для эмпирических исследований есть достаточно надежная база, которая позволит получать согласующиеся друг с другом результаты и делать на их основании выводы: о месте идеологии качества с учетом организационных реалий нашей страны, о целесообразности введения и/или сертификации СМК, об отношении аналитиков к подобным мероприятиям. Так или иначе, в основе этих исследований должно лежать понимание идеологии TQM, основы которой мы постарались систематизировать в этой статье.

\section{Список литературы}

1. Глудкин О.П., Горбунов Н.М., Гуров А.И, Зорин Ю.В. Всеобщее управление качеством: Учебник для вузов. М.: Радио и связь, 1999.

2. Голубицкий С. Отчим качества // Бизнес-журнал (изд. дом «Компьютера»). 2005. № 1.

3. Нив Г. Пространство доктора Деминга. М.: Альпина Бизнес Букс.

4. Ребрин Ю.И. Управление качеством: Учебное пособие. ТРТУ. Таганрог, 1998.

5. Рубаник Ю.Т. 14 пунктов Деминга - программа действий для выживания производителей России // Стандарты и качество. 1995. № 7.

6. Рубаник Ю.Т. Российские корпорации выбирают траекторию организационного 
развития // Бизнес-образование. 2004. № 6.

7. Рубаник Ю.Т. Путь качества как национальная идея для России. URL: http://www.deming.ru.

8. Шанк Дж., Говиндараджан В. Стратегическое управление затратами: новые методы увеличения конкурентоспособности. С.-Пб: Бизнес-микро, 1999.

9. Australian Manufacturing Council Study, AMC Study (1992), Leading the Way: A study of Best Manufacturing Practices in Australia and New Zealand. (1992) 59-63.

10. Arnold, K.L. (1994), The manager's guide to ISO 9000. NY: The Free Press.

11. Beattie, K.R., and Sohal, A.S. (1999), Implementing ISO 9000: A study of its benefits among Australian organizations. Total Quality Management, 10(1) (1999) 95-106.

12. Brooks, I. (1995), The yellow brick road: The path to building a quality business in New Zealand. Auckland: Nahanni Publishing.

13. Brown, A., and Van der Wiele, T. (1995), Industry experience with ISO 9000, Asia Pacific Journal of Quality Management, 4(2) (1995) 8-17.

14. Buttle, F. (1997), ISO 9000: Marketing motivations and benefits, International Journal of Quality and Reliability Management, 14(9) (1997) 936-947.

15. Carr, S., Mak, Y.T., and Needham, J.E. (1997), Differences in strategy, quality management practices and performance reporting systems between ISO accredited and non-ISO accredited companies, Management Accounting Research, 8 (1997) 383-384.

16. Chittenden, F., Poutziouris, P., and Muhktar, S.M. (1998), Small firms and ISO 9000 approach to quality management, International Small Business Journal, 17(1) (1998) 73- 88.

17. Dale, B.G. (1994), Managing quality. Hertfordshire, UK: Prentice Hall International.

18. Easton, G.S., and Jarrell, S.L. (1998), The effects of total quality management on corporate performance: An empirical investigation, Journal of Business, 71(2) (1998) 253-307.

19. Elmuti, D., and Kathawala, Y. (1997), An investigation into the effects of ISO 9000 on participants' attitudes and job performance, Production and Inventory Management Journal, 38(2) (1997) 52-57.

20. Finley, L., and Buntzman, G. F. (1994), What does affect company performance?, Arkansas Business and Economic Review, 27(2) (1994) 1.

21. Haversjo, T. (2000), The financial effects of ISO 9000 registration for Danish companies, Managerial Auditing Journal, 15(1/2) (2000) 47- 52.

22. Heras, I., Casadesus, M., and Dick, G. P. M. (2002), ISO 9000 certification and the bottom line: A comparative study of the profitability of Basque region companies, Managerial Auditing Journal, 17(1/2) (2002) 72-78.

23. Heras, I., Dick, G. P. M., and Casadesus, M. (2002), ISO registration's impact on sales and profitability: A longitudinal analysis of performance before and after accreditation, International Journal of Quality and Reliability Management, 19(6) (2002) 774-791.

24. Hua, H., Chin, K.S., Sun, H., and Xu, Y. (2000), An empirical study on quality management practices in Shanghai manufacturing industries, Total Quality Management, 11(8) (2000) $1111-1122$.

25. International Organization for Standardization. Tenth Cycle. The ISO Survey of ISO 9000 and ISO 14000 Certificates. International Organization for Standardization, Geneva.

26. ISO (1987), Original 1987 bulletin from the International Organization for Standardization.

27. ISO (2009), The ISO Survey of Certifications 2008.

28. Juran, J.M. (1999), Juran urges research: What they're saying about standards, Quality Progress, 31 (1999).

29. Lisiecka, K. (1999), ISO 9000 standards and TQM strategy-business improvement tools for Polish companies, Managerial Auditing Journal, 14(1/2) (1999) 40- 43.

30. Low, S.P., Tan, B.K., and Ang, A.L. (1999), Effectiveness of ISO 9000 in raising construction quality standards: Some empirical evidence using CONQUAS score, Structural Survey, 17(2) (1999) 89.

31. Lloyds Register Quality Assurance Limited (1993), Setting Standards for Better Business, 
Report of Survey findings. London, UK.

32. Maddala, G.S. (1977), Self-selectivity problems in econometric models. In P. R. Krishnaiah (Ed.), Application of statistics. New York, NY: Elsevier.

33. Maddala, G.S. (1983), Limited dependent and qualitative variables in econometrics. Econometric Society Monographs in Quantitative Economics, vol. 3. New York, NY: Cambridge University Press.

34. Marquardt, D.W. (1992), ISO 9000: A universal standard of quality, Management Review, 81(1) (1992) 50- 52.

35. Mas-Colell, A., Whinston, M.D., Green, J.R. (1995), Microeconomic Theory. Oxford, New York, NY: Oxford University Press.

36. McAdam, R., and McKeown, M. (1999), Life after ISO: An analysis of the impact of ISO 9000 and total quality management on small businesses in Northern Ireland, Total Quality Management, 10 (1999) 229-241.

37. Nagar, V., and Rajan, M. (2001), The revenue implications of financial and operational measures of product quality, The Accounting Review, 76(4) (2001) 495-513.

38. Nicolau, J.L., Sellers, R. (2002), The stock market's reaction to quality certification: Empirical evidence from Spain, European Journal of Operational Research, 142 (2002) 632641.

39. Peach, R.W. (1997), The ISO 9000 handbook. Chicago: Irwin Professional Publishing.

40. Quazi, H.A., Chang, W.H., and Chan, T.M. (2002), Impact of ISO 9000 certification on quality management practices: A comparative study, Total Quality Management, 13(1) (2002) 53-67.

41. Rao, S.S., Ragu-Nathan, T.S., and Solis, L.E. (1997), Does ISO 9000 have an effect on quality management practices? An international empirical study, Total Quality Management, 8(6) (1997) 335- 346.

42. Rayner, P., and Porter, L.J. (1991), BS 5750/ISO 9000-The experience of small and medium-sized firms, International Journal of Quality and Reliability Management, 8(6) (1991) 16-28.

43. Reed, R., Lemak, D., and Montgomery, J.C. (1996, January), Beyond process: TQM content and firm performance, Academy of Management Review 1 (1996).

44. Shams-ur, R. (2001), A comparative study of TQM practice and organizational performance of SMEs with and without ISO 9000 certification, International Journal of Quality and Reliability Management, 18(1) (2001) 35- 49.

45. Sharma, D.S. (2005), The association between ISO 9000 certification and financial performance, The International Journal of Accounting, 40 (2005) 151- 172.

46. Simmons, B. and L., and White, M.A. (1999), The relationship between ISO 9000 and business performance: Does registration really matter?, Journal of Managerial Issues, 11(3) (1999) 330-343.

47. Sun, H. (1999), Diffusion and contribution of the total quality management: An empirical study in Norway, Total Quality Management, 10 (1999) 901-914.

48. Terlaak, A., King, A.A. (2006), The effect of certification with the ISO 9000 Quality Management Standard: A signaling approach, Journal of Economic Behavior \& Organization, 60 (2006) 579-602.

49. Terziovski, M., Power, D., Sohal, A. (2003), The longitudinal effects of the ISO 9000 certification process on business performance, European Journal of Operational Research, 146 (2003) 580-595.

50. Wiele, T.V.D., \& Brown, A. (1997-98), Venturing down the TQM path for SME's, International Small Business Journal, 16 (1998) 50- 68.

51. Yamada, S. (2001), Economical aspects of ISO 9000 certification in Japanese companies. Quality Congress, Annual Quality Congress Proceedings (pp. 647- 659). Milwaukee: American Society for Quality. 